Tropical Journal of Pharmaceutical Research June 2016; 15 (6): 1327-1334

ISSN: $1596-5996$ (print); 1596-9827 (electronic) (C) Pharmacotherapy Group, Faculty of Pharmacy, University of Benin, Benin City, 300001 Nigeria. All rights reserved.

\title{
Prevalence of chronic diseases in private healthcare sector of South Africa: A threat to public health
}

\author{
Lourens Johannes Rothmann ${ }^{1}$, Martha Susanna Lubbe ${ }^{1}$, Jan Hendrik Philippus \\ Serfontein ${ }^{1}$, Jan Jakobus Gerber ${ }^{1}$ and Madeeha Malik ${ }^{1,2 *}$ \\ ${ }^{1}$ Medicine Usage in South Africa (MUSA), School of Pharmacy, Faculty of Health Sciences, North-West University, \\ Potchefstroom Campus, Potchefstroom, South Africa, ${ }^{2}$ Hamdard Institute of Pharmaceutical Sciences, Hamdard University, \\ Islamabad, Pakistan
}

*For correspondence: Email: madeehamalik15@gmail.com; Tel: 0027793624335

Received: 13 September $2015 \quad$ Revised accepted: 12 May 2016

\begin{abstract}
Purpose: To evaluate the prevalence of patients suffering from registered chronic disease list (CDL) conditions in a section of the South African private health sector from 2008 - 2012.

Methods: This study was a retrospective analysis of the medicine claims database of a nationally (South African) representative Pharmacy Benefit Management (PBM) company data between 2008 and 2012. Statistical analysis was used to analyse the data. Descriptive analysis was performed to calculate the prevalence of $C D L$ conditions for the entire population, and stratified by age and gender. However, MIXED linear modelling was used to determine changes in the average number of CDL conditions per patient, adjusted for age and gender from 2008 - 2012.

Results: An increase of 0.20 in chronic diseases was observed from 2008 - 2012 in patients having any $C D L$ condition, with an average of $1.57(1.57-1.58,95 \%$ Cl) co-morbid CDL conditions in 2008 and $1.77(1.77-1.78,95 \% \mathrm{Cl})$ in 2012. This increase in average number of CDL conditions per patient between 2008 and 2012 was statistically significant $(p<0.05)$, but with no large practical significance (d $<0.8)$.

Conclusion: Prevalence of patients with CDL conditions along with risk of co-morbidity has been increasing with time in the private health sector of South Africa. Risk of increased co-morbidity with age and among different genders was prevalent.
\end{abstract}

Keywords: Chronic disease list, Prevalence, Private health sector, Co-morbidity

Tropical Journal of Pharmaceutical Research is indexed by Science Citation Index (SciSearch), Scopus, International Pharmaceutical Abstract, Chemical Abstracts, Embase, Index Copernicus, EBSCO, African Index Medicus, JournalSeek, Journal Citation Reports/Science Edition, Directory of Open Access Journals (DOAJ), African Journal Online, Bioline International, Open-J-Gate and Pharmacy Abstracts

\section{INTRODUCTION}

Chronic non-communicable diseases (NCDs) such as diabetes, cardiovascular disease (CVD) and cancers pose a major public health threat worldwide. According to an estimate by $\mathrm{WHO}$ by 2020 , one-third of the global burden of disease will be attributable to chronic diseases [1]. Nearly one-third and $44 \%$ of the deaths before age 60 are due to chronic diseases in middle-income and low income countries, respectively. Various risk factors including unhealthy diets, physical inactivity, alcohol consumption, poor knowledge of health workers, low quality services and lack of access to medicines have been identified towards contribution of burden of chronic diseases [2]. This rise of burden of chronic diseases in turn has led to high costs of illness and potentially significant productivity losses [3].

The burden of non-communicable diseases as well as multi-morbidity, the co-existence of more 
than one chronic condition in one person, is particularly rising in Africa. According to the study conducted by The Global Burden of Disease, HIV ranks first, TB fourth, cerebrovascular disease seventh, diabetes eighth while noncommunicable diseases contribute to fifty percent causes of morbidity in South Africa [4]. An estimated increase from 11 to $13 \%$ and 3.2 to $4.0 \%$ since 2009 to 2025 , for CDL conditions and multi-morbidity is anticipated [4]. The current situation of increased burden of chronic diseases is a major challenge to existing models of healthcare delivery systems in South Africa. Thus, it is important to recognise such illnesses and strategize appropriately to address them at individual patient care level [5].

To address this dilemma, the South African Council for Medical Schemes formulated a chronic disease list (CDL) including the most common, life-threatening diseases. These conditions fall under a larger group, called prescribed minimum benefits, defined as a set of benefits to ensure that all medical scheme members have access to certain minimum health services; regardless of the benefit option they have selected $[6,7]$. This has created a positive impact on prescribing practices carried in private healthcare facilities. But, still limited data exists on assessing the prevalence of these particular combinations or clusters of chronic conditions, as most of the studies conducted so far in South Africa have been focused on comorbidities of a specific disease.

Thus, the present study was designed to evaluate the prevalence of patients suffering from registered chronic disease list (CDL) conditions in a section of the South African private health sector from 2008 - 2012.

\section{EXPERIMENTAL}

This study was a retrospective analysis of the medicine claims database of a nationally (South African) representative PBM company data between 2008 and 2012.Approval was obtained for the study from the appropriate Pharmacy Benefit Management companies (PBM) as well as from the Ethics Committee of the North-West University (Human, Potchefstroom campus) (no. NWU-0046-08-A5) to conduct this drug utilisation study. The PBM Company provides services to four capitation providers, thirty-two medical schemes and four sick benefit society funds. The medicine benefits of approximately 1.6 million beneficiaries are maintained annually by the PBM company.
The data fields used in this study include: quantity of medication claimed, member gender, member date of birth, NAPPI code, ICD-10 code, diagnose code, active pharmacological ingredient of the medication, number of prescriptions dispensed containing medication used to treat CDL conditions and period in which the item was dispensed. The chronic conditions included in this paper are listed (Table 1).

Table 1: South African chronic disease list (CDL)

\begin{tabular}{ll}
\hline Addison's disease & Dysrhythmia \\
Asthama & Epilepsyl \\
Bronchiectasis & Glaucoma \\
Cardiac failure & Haemophilia A \\
Cardiomyopathy & Haemophilia B \\
Chronic obstructive pulmonary & Hyperlipidemia \\
disease & \\
Chronic renal disease & Hypertension \\
Coronoary artery disease & Hypothyrodisim \\
Crohn's disease & Multiple sclerosis \\
Diabetes insipudus & Parkinson's disease \\
Diabetes mellitus type I & Rheumatoid arthritis \\
Diabetes mellitus type II & Schizophrenia \\
Systemic lupus erythematosus & Ulcerative colitis \\
\hline
\end{tabular}

Analyses were performed on patient level. The prevalence of each condition was calculated by dividing the number of patients with the condition by the number of active patients. Statistical Analysis System®, SAS 9.3®and Statistical Package for the Social Sciences (IBM SPSS $\mathbb{R}$ 22) were used to analyse the data. Descriptive analysis was performed for calculating the prevalence of CDL conditions for the entire population and stratified by age and gender. MIXED linear modelling was used to calculate average number of CDL conditions per patient for the influence of age and gender differences so that the same patients prevalent in 2008 remained also prevalent in 2012. These also involved patients that have either entered or left the medical scheme for any reason or came into the medical scheme any time during the study period under consideration.

\section{RESULTS}

\section{Prevalence of patients with at least one CDL condition}

The total number of patients on the database were 974,497 patients in $2008,1,307,528$ in 2009, 1,220,289 in 2010, 1,077,834 in 2011 and $1,029,699$ patients in 2012. Patients with at least one CDL condition represented $21.9 \%$ ( $n=214$ $279)$ of the total number of patients on the database in 2008, and they increased to $26 \%$ ( $=268685$ ) in 2012. The representation of female 
patients with at least one CDL condition increased from $23.3 \%$ (2008) to $26.9 \%$ (2012) and those of male patients from $20.3 \%$ (2008) to $25.1 \%$ (2012). There was an increase in the number of patients with CDL conditions across all age groups except for age group $\leq 12$ year with a decrease from $2.9 \%$ (2008) to $2.7 \%$ (2012). The other age groups: $12>$ age $\leq 50$ years increased from $9.4 \%(2008)$ to $10.7 \%$ (2012); $50>$ age $\leq 60$ years increase from 33.6 $\%$ (2008) to $40.1 \%$ (2012); $60>$ age $\leq 70$ years increased from $57.1 \%(2008)$ to $65 \%$ (2012) and age $>70$ years from $71.5 \%$ in 2008 to 77.8 $\%$ in 2012. A detail summary is given (Table 2 ).

The estimated annual increase was also higher for male patients and their associated CDL conditions. All age groups had their highest prevalence in 2012, except for age groups less than 12 and 12 to 50 years. Age group > 70 years had the highest prevalence of patients in all the age groups for the study period in 2012 with $29.3 \%(n=78$ 879). The largest increase in the number of patients with at least one CDL condition were between 2008 and 2012 was for age group $>70$ years $(36.2 \% ; n=20985)$ and for chronic conditions (group > 70 years; $51.7 \%$; $n=55613$ ). Age group $>70$ years, thus had the highest estimated annual patient increase of $9 \%$ ( $n=5$ 246) and estimated increase in number of CDL conditions of $12.9 \%(n=13$ 903) (Table 3).

Of all the CDL conditions, hypertension had the highest contribution during each of the study years followed by hyperlipidaemia. All 26 conditions, had a patient increase of 139336 with an estimated annual increase of 34834 . The total number of patients increased by $41.3 \%$ ( $n=$ 139 336) between 2008 and 2012 with an estimated annual increase of $10.3 \%(n=34$ 834). All CDL conditions except bronchiectasis had an overall increase in the number of patients from 2008 until 2012, hypertension had the highest increase ( $n=49531)$, followed by hyperlipidaemia $(\mathrm{n}=35490)$ and diabetes mellitus type $2(n=17120)$. Bronchiectasis had an overall decrease between 2008 and 2012 of 38 patients. The prevalence included patients with a single CDL condition or in combination with other CDL conditions. Diabetes insipidus had the lowest patient increase $(n=3)$ between 2008 and 2012 (Table 4).

Changes in the number of CDL conditions, adjusted for influence of age and gender

An increase of 0.20 in chronic diseases was observed since 2008-2012 in patients having any
CDL condition with an average of 1.57 (2008) comorbid CDL conditions to 1.77 in 2012. This difference in increase in average number of $\mathrm{CDL}$ conditions per patient between 2008 and 2012 was of statistical significance $(p<0.05)$, but no large practical significance $(\mathrm{d}<0.8)$ (Table 5).

All of the CDL conditions had increases in the average number of co-morbid CDL conditions per patient: however, glaucoma, haemophilia B and bronchiectasis were the only CDL conditions with an increase in co-morbid CDL conditions per patient of large practical significance $(d>0.8)$. Asthma (increased by 0.18 co-morbid CDL conditions), hyperlipidaemia (increased by 0.20 ), hypertension (increased by 0.16 ), hypothyroidism (increased by 0.67 ) and diabetes mellitus type 2 (increased by 0.24). Addison's disease (increased by 0.23 ), coronary artery disease (increased by 0.25 ), cardiac failure (increased by 0.14 ), multiple sclerosis (increased by 0.92), Parkinson's disease (increased by 0.25 ) and chronic obstructive pulmonary disease increased by $0.17 \mathrm{CDL}$ conditions per patient between 2008 and 2012. The CDL condition with the smallest increase in CDL conditions per patient was for schizophrenia with 0.08 and the highest increase for patients with bronchiectasis with 1.07 CDL conditions per patient.

Epilepsy, glaucoma, haemophilia $A$ and haemophilia $B$ had increases of $0.17,0.15,0.29$ and 0.50 respectively between 2008 and 2012 . The only CDL condition with an overall decrease of 0.21 in average number of CDL conditions was chronic renal disease. The CDL condition with the highest number of co-morbid CDL conditions was chronic renal disease with 3.54 in 2008 to 3.37 in 2011. Cardiomyopathy had the highest average number of CDL conditions per patient during 2012 with 3.36. Average number of co-morbidities with hypothyroidism had an overall increase from 2008 with 1.67 to 2012 with 2.34.

\section{DISCUSSION}

Chronic Diseases List conditions play a vital role in the consumption of health resources, not only due to the number of patients increasing but also the financial impact on medical schemes, patients and other resources. The present study reported continuous increase of total number of patients with at least one CDL over time. This might be attributed to chronic disease related lifestyle risk factors, increased registration of CDL conditions by medical scheme beneficiaries and increase in the number of medical scheme 
Table 2: Prevalence of patients with at least one CDI condition according to the total number of patients on the database

\begin{tabular}{|c|c|c|c|c|c|c|c|}
\hline Description & & & 2008 & 2009 & 2010 & 2011 & 2012 \\
\hline \multirow{7}{*}{$\begin{array}{c}\text { Total number of } \\
\text { patients } \\
\text { On the database }(n)\end{array}$} & \multirow{3}{*}{ Gender } & Female & 538254 & 712305 & 661007 & 578593 & 545068 \\
\hline & & Male & 436243 & 595223 & 559282 & 499237 & 484627 \\
\hline & & $>12$ & 124440 & 192387 & 175664 & 156805 & 146296 \\
\hline & \multirow{4}{*}{$\begin{array}{l}\text { Age Group } \\
\text { (Years) }\end{array}$} & $\leq 12->50$ & 524962 & 705064 & 640480 & 557250 & 523587 \\
\hline & & $\leq 50->60$ & 154659 & 191661 & 182559 & 160018 & 156430 \\
\hline & & $\leq 60->70$ & 89570 & 115114 & 114697 & 102425 & 102047 \\
\hline & & $\leq 70$ & 80866 & 103302 & 106889 & 101336 & 101339 \\
\hline \multirow{11}{*}{$\begin{array}{c}\text { Total number of } \\
\text { patients } \\
\text { With at least one CDL } \\
\text { Condition ( } n)\end{array}$} & Total & & 974497 & 1307528 & 1220289 & 1077834 & 1029699 \\
\hline & \multirow{3}{*}{ Gender } & Female & $125548(23.3 \%)$ & $142690(20 \%)$ & $140133(21.2 \%)$ & $129192(22.3 \%)$ & $146678(26.9 \%)$ \\
\hline & & Male & $88731(20.3 \%)$ & $112244(18.8 \%)$ & $111062(19.8 \%)$ & $104159(20.8 \%)$ & $122007(25.1 \%)$ \\
\hline & & $>12$ & $3611(2.9 \%)$ & $4511(2.3 \%)$ & $2273(2.4 \%)$ & $4165(2.6 \%)$ & $4088(2.7 \%)$ \\
\hline & \multirow{3}{*}{$\begin{array}{l}\text { Age Group } \\
\text { (Years) }\end{array}$} & $\leq 12->50$ & $49519(9.4 \%)$ & $60697(8.6 \%)$ & $57516(8.9 \%)$ & $52628(2.6 \%)$ & $56479(10.7 \%)$ \\
\hline & & $\begin{array}{l}\leq 50->60 \\
\leq 60->70\end{array}$ & $\begin{array}{l}52040(33.6 \%) \\
51215(57.1 \%)\end{array}$ & $\begin{array}{l}61735(32.2 \%) \\
60026(52.1 \%)\end{array}$ & $\begin{array}{c}60167(32.9 \%) \\
59716(52 \%)\end{array}$ & $\begin{array}{c}54364(9.4 \%) \\
54988(33.9 \%)\end{array}$ & $\begin{array}{l}62851(40.1 \%) \\
66388(65 \%)\end{array}$ \\
\hline & & $\leq 70$ & $57894(71.5 \%)$ & $67965(65.7 \%)$ & $69523(65 \%)$ & $67206(53.6 \%)$ & $78879(77.8 \%)$ \\
\hline & \multirow{2}{*}{ Total } & & $214279(21.9 \%)$ & $254934(19.4 \%)$ & $251195(20.5 \%)$ & $233351(21.6 \%)$ & $268685(26 \%)$ \\
\hline & & Female & 9893928 & 12834715 & 12103038 * & 10327159 * & 9237012 * \\
\hline & \multirow{2}{*}{ Gender } & Male & 6545325 & 8814276 & 8424739 * & 7439420 * & 7172238 * \\
\hline & & $>12$ & 1085511 & 1727830 & 1552825 & 1303782 & 1209684 \\
\hline \multirow{8}{*}{$\begin{array}{l}\text { Total number of items } \\
\text { on the database }(n)\end{array}$} & \multirow{4}{*}{$\begin{array}{l}\text { Age Group } \\
\text { (Years) }\end{array}$} & $\leq 12->50$ & 6416499 & 8347338 & 7604671 & 6378677 & 5906537 \\
\hline & & $\leq 50->60$ & 3288346 & 4153722 & 3945088 & 3384147 & 3280626 \\
\hline & & $\leq 60->70$ & 2585064 & 3418324 & 3366712 & 2945546 & 2876437 \\
\hline & & $\leq 70$ & 3063833 & 4001777 & 4058470 & 3754442 & 3136008 \\
\hline & Total & & 16439253 & 21648991 & 20527766 & 17766594 & 16409292 \\
\hline & \multirow{3}{*}{ Gender } & Female & 1376575 (13.9 \%) & $1804555(14 \%)$ & $1728965(14.9 \%)$ & $\begin{array}{c}1735386(16.8 \\
\%)\end{array}$ & $\begin{array}{c}1551209(16.7 \\
\%)\end{array}$ \\
\hline & & Male & $1174733(17.9 \%)$ & $1526551(17.3 \%)$ & $1480315(17.5 \%)$ & $\begin{array}{c}1515603(20.3 \\
\%)\end{array}$ & $\begin{array}{c}1513911(21.1 \\
\%)\end{array}$ \\
\hline & & $>12$ & $20380(1.8 \%)$ & $43014(2.4 \%)$ & $39469(2.5 \%)$ & 40356 (3 \%) & $34964(2.8 \%)$ \\
\hline \multirow{4}{*}{$\begin{array}{l}\text { Total number of CDL } \\
\text { conditions items }(n)\end{array}$} & \multirow{3}{*}{$\begin{array}{l}\text { Age Group } \\
\text { (Years) }\end{array}$} & $\leq 12->50$ & $445947(6.9 \%)$ & $578377(6.9 \%)$ & $541606(7.1 \%)$ & $554426(8.6 \%)$ & $520912(8.8 \%)$ \\
\hline & & $\begin{array}{l}\leq 50->60 \\
\leq 60->70\end{array}$ & $\begin{array}{c}589949(17.9 \%) \\
646249(25 \%)\end{array}$ & $\begin{array}{l}746537(17.9 \%) \\
837411(24.5 \%)\end{array}$ & $\begin{array}{c}710961(18 \%) \\
807093(23.9 \%)\end{array}$ & $\begin{array}{c}712964(21 \%) \\
817432(27.7 \%)\end{array}$ & $\begin{array}{l}712071(21.7 \%) \\
822188(28.5 \%)\end{array}$ \\
\hline & & $\leq 70$ & $848783(27 \%)$ & $1125767(28.1 \%)$ & $1110151(27.3 \%)$ & $\begin{array}{c}1124811(29.9 \\
\%)\end{array}$ & $974985(31 \%)$ \\
\hline & Total & & 2551308 (15.5\%) & 3331106 (15.3\%) & 3209280 (15.6\%) & $\begin{array}{c}3249989 \text { (18.2 } \\
\%)\end{array}$ & $\begin{array}{c}3065120(18.6 \\
\%)\end{array}$ \\
\hline
\end{tabular}

Note: All percentages are expressed as a percentage of the equivalent group on the total database. ${ }^{*}$ (2010)- Unidentified patients ( $\mathrm{n}=11$; R1 045.66), (2011)- Unidentified patients ( $n=15$; R316.05), (2012- Unidentified patients ( $n=42$; R1 334.97) 
Table 3: Change in number of patients with at least one CDL condition and the total number of CDL conditions, stratified according to age and gender

\begin{tabular}{|c|c|c|c|c|c|c|c|c|c|}
\hline & & \multicolumn{2}{|c|}{2008} & \multicolumn{2}{|c|}{2012} & \multirow{2}{*}{$\begin{array}{l}\text { Patient change } \\
\text { (2008 vs 2012) } \\
\text { (n) }\end{array}$} & \multirow{2}{*}{$\begin{array}{c}\text { Chronic disease } \\
\text { change (2008 vs } \\
2012) \\
(n)\end{array}$} & \multirow{2}{*}{$\begin{array}{l}\text { Estimated annual } \\
\text { patient change } \\
\text { (n) }\end{array}$} & \multirow{2}{*}{$\begin{array}{l}\text { Estimated annual } \\
\text { chronic disease } \\
\text { change (n) }\end{array}$} \\
\hline \multicolumn{2}{|c|}{ Description } & Patients & $\begin{array}{l}\text { Chronic } \\
\text { diseases }\end{array}$ & Patients & $\begin{array}{l}\text { Chronic } \\
\text { diseases }\end{array}$ & & & & \\
\hline $\begin{array}{c}\text { Age Group } \\
\text { (Years) }\end{array}$ & 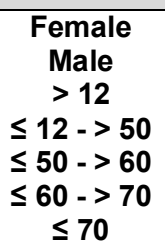 & $\begin{array}{c}125548 \\
88731 \\
3611 \\
49519 \\
52040 \\
51215 \\
57894\end{array}$ & $\begin{array}{c}193182 \\
144200 \\
3654 \\
63090 \\
77881 \\
85375 \\
107382\end{array}$ & $\begin{array}{c}146678 \\
122007 \\
4088 \\
56479 \\
62851 \\
66388 \\
78879\end{array}$ & $\begin{array}{c}258263 \\
218455 \\
4137 \\
77362 \\
105427 \\
126797 \\
162995\end{array}$ & $\begin{array}{c}\uparrow 21130(16.8 \%) \\
\uparrow 33276(37.5 \%) \\
\uparrow 477(13.2 \%) \\
\uparrow 6960(14 \%) \\
\uparrow 10811(17.2 \%) \\
\uparrow 15173(29.6 \%) \\
\uparrow 20985(36.2 \%)\end{array}$ & $\begin{aligned} & \uparrow \\
\uparrow & 081(33.6 \%) \\
\uparrow & 74255(51.4 \%) \\
& \uparrow 483(13.2 \%) \\
& 14272(22.6 \%) \\
\uparrow & 27546(35.3 \%) \\
\uparrow & 41422(48.5 \%) \\
\uparrow & 55613(51.7 \%)\end{aligned}$ & $\begin{array}{c}\uparrow 5283(4.2 \%) \\
\uparrow 8319(9.3 \%) \\
\uparrow 119(3.3 \%) \\
\uparrow 1740(3.5 \%) \\
\uparrow 2703(4.3 \%) \\
\uparrow 3793(7.4 \%) \\
\uparrow 5246(9 \%)\end{array}$ & $\begin{array}{c}\uparrow 16270(8.4 \%) \\
\uparrow 18564(12.8 \%) \\
\uparrow 121(3.3 \%) \\
\uparrow 3568(5.6 \%) \\
\uparrow 6887(8.8 \%) \\
\uparrow 10356(12.1 \%) \\
\uparrow 13903(12.9 \%)\end{array}$ \\
\hline
\end{tabular}

Table 4: Prevalence of patients with all individual CDL conditions per condition

\begin{tabular}{|c|c|c|c|c|c|c|c|c|c|c|c|c|}
\hline \multirow{2}{*}{ CHRONIC DISEASE } & \multicolumn{2}{|c|}{2008} & \multicolumn{2}{|c|}{2009} & \multicolumn{2}{|c|}{2010} & \multicolumn{2}{|c|}{2011} & \multicolumn{2}{|c|}{2012} & \multirow{2}{*}{$\begin{array}{l}\text { Change } \\
\text { (2008 vs } \\
2012)(n)\end{array}$} & \multirow{2}{*}{$\begin{array}{c}\text { Estimated } \\
\text { annual } \\
\text { change } \\
\text { (n) }\end{array}$} \\
\hline & $\begin{array}{l}\text { Patients } \\
\text { (n) }\end{array}$ & $\%$ & $\begin{array}{l}\text { Patients } \\
\text { (n) }\end{array}$ & $\%$ & $\begin{array}{l}\text { Patients } \\
\text { (n) }\end{array}$ & $\%$ & $\begin{array}{l}\text { Patients } \\
\text { (n) }\end{array}$ & $\%$ & $\begin{array}{l}\text { Patients } \\
\text { (n) }\end{array}$ & $\%$ & & \\
\hline Hypertension & 57627 & 44.4 & 70058 & 50.1 & 69193 & 50.7 & 62909 & 50.9 & 69248 & 50.8 & $\begin{array}{l}\uparrow 11621 \\
(20.1 \%) \\
\downarrow 12834\end{array}$ & $\begin{array}{c}\uparrow 2905(5 \%) \\
\downarrow 3209\end{array}$ \\
\hline Hypothyroidism & 24558 & 18.9 & 11824 & 8.4 & 11899 & 8.7 & 10658 & 8.6 & 11724 & 8.6 & $\begin{array}{l}(52.2 \%) \\
\uparrow 2977\end{array}$ & $(13 \%)$ \\
\hline Hyperlipidaemia & 15239 & 11.7 & 18385 & 13.1 & 17778 & 13 & 15002 & 12.1 & 18216 & 13.3 & $\begin{array}{l}(12.1 \%) \\
\uparrow 1664\end{array}$ & $\uparrow 744(3 \%)$ \\
\hline Asthma & 12139 & 9.3 & 14559 & 10.4 & 14107 & 10.3 & 13586 & 10.9 & 13803 & 10.1 & $\begin{array}{c}(13.7 \%) \\
\uparrow 975\end{array}$ & $\uparrow 416(3.4 \%)$ \\
\hline Diabetes Mellitus Type 2 & 6158 & 4.74 & 7425 & 5.3 & 7097 & 5.2 & 6393 & 5.1 & 7133 & 5.2 & $\begin{array}{c}(15.8 \%) \\
\uparrow 895\end{array}$ & $\uparrow 244(3.9 \%)$ \\
\hline Epilepsy & 3770 & 2.9 & 4991 & 3.5 & 4635 & 3.4 & 4101 & 3.3 & 4665 & 3.4 & $\begin{array}{l}(23.7 \%) \\
\downarrow 1256\end{array}$ & $\begin{array}{c}\uparrow 224(5.9 \%) \\
\downarrow 314\end{array}$ \\
\hline Diabetes Mellitus Type 1 & 2912 & 2.2 & 2199 & 1.5 & 1744 & 1.2 & 1952 & 1.5 & 1656 & 1.2 & $\begin{array}{c}(43.1 \%) \\
\uparrow 563\end{array}$ & $(10.7 \%)$ \\
\hline Glaucoma & 1622 & 1.2 & 1967 & 1.4 & 1987 & 1.4 & 1817 & 1.4 & 2185 & 1.6 & $\begin{array}{c}(34.7 \%) \\
\uparrow 956\end{array}$ & $\begin{array}{c}\uparrow 141(8.6 \%) \\
\uparrow 239\end{array}$ \\
\hline Cardiac failure & 1271 & 0.9 & 2402 & 1.7 & 2277 & 1.6 & 1861 & 1.5 & 2227 & 1.6 & $\begin{array}{c}(75.2 \%) \\
\uparrow 385\end{array}$ & $(18.8 \%)$ \\
\hline Rheumatoid arthritis & 872 & 0.6 & 1109 & 0.7 & 1108 & 0.8 & 1042 & 0.8 & 1257 & 0.9 & $(44.1 \%)$ & $\uparrow 96(11 \%)$ \\
\hline Dysthyrthmia & 759 & 0.5 & 902 & 0.6 & 900 & 0.6 & 809 & 0.6 & 846 & 0.6 & $\uparrow 87(11.4 \%)$ & $\uparrow 22(2.8 \%)$ \\
\hline
\end{tabular}

Trop J Pharm Res, June 2016; 15(6): 1331 
Rothmann et al

\begin{tabular}{|c|c|c|c|c|c|c|c|c|c|c|c|c|}
\hline Cardiomyopathy & 606 & 0.4 & 474 & 0.3 & 416 & 0.3 & 349 & 0.2 & 270 & 0.2 & $\begin{array}{c}\downarrow 336 \\
(55.4 \%)\end{array}$ & $\begin{array}{c}\downarrow \downarrow 84 \\
(13.8 \%)\end{array}$ \\
\hline Coronary artery disease & 588 & 0.4 & 781 & 0.5 & 641 & 0.4 & 991 & 0.8 & 562 & 0.3 & $\downarrow 26(4.4 \%)$ & $\downarrow 7(1.1 \%)$ \\
\hline Parkinson's disease & 531 & 0.4 & 645 & 0.4 & 642 & 0.4 & 568 & 0.4 & 598 & 0.4 & $\uparrow 67(12.6 \%)$ & $\uparrow 17(3.1 \%)$ \\
\hline $\begin{array}{l}\text { Chronic obstructive pulmonary } \\
\text { disease }\end{array}$ & 421 & 0.3 & 578 & 0.4 & 538 & 0.3 & 451 & 0.3 & 504 & 0.3 & $\uparrow 83(19.7 \%)$ & $\uparrow 21(4.9 \%)$ \\
\hline Crohn's disease & 100 & 0.0 & 239 & 0.1 & 232 & 0.1 & 218 & 0.1 & 237 & 0.1 & $\begin{array}{c}\uparrow 137(137 \%) \\
\uparrow 108\end{array}$ & $\begin{array}{c}\uparrow 34 \\
(34.2 \%) \\
\uparrow 27\end{array}$ \\
\hline Multiple sclerosis & 63 & 0.05 & 315 & 0.2 & 293 & 0.22 & 198 & 0.16 & 171 & 0.13 & $(171.4 \%)$ & $(42.8 \%)$ \\
\hline Ulcerative colitis & 42 & 0.03 & 92 & 0.07 & 77 & 0.06 & 71 & 0.06 & 84 & 0.06 & $\uparrow 42(100 \%)$ & $\uparrow 11(25 \%)$ \\
\hline Addison's disease & 40 & 0.03 & 42 & 0.03 & 36 & 0.03 & 37 & 0.03 & 38 & 0.03 & $\downarrow 2(5 \%)$ & $\downarrow 1(1.2 \%)$ \\
\hline Systemic lupus erythematosus & 38 & 0.03 & 51 & 0.04 & 59 & 0.04 & 49 & 0.04 & 65 & 0.05 & $\uparrow 27(71 \%)$ & $\uparrow 7(17.7 \%)$ \\
\hline Bronchiectasis & 36 & 0.03 & 36 & 0.03 & 30 & 0.02 & 21 & 0.02 & 4 & 0.00 & $\downarrow 32(88.8 \%)$ & $\downarrow 8(22.2 \%)$ \\
\hline Haemophilia A & 20 & 0.02 & 27 & 0.02 & 31 & 0.02 & 35 & 0.03 & 30 & 0.02 & $\uparrow 10(50 \%)$ & $\uparrow 3(12.5 \%)$ \\
\hline Diabetes insipidus & 10 & 0.01 & 7 & 0.01 & 10 & 0.01 & 8 & 0.01 & 8 & 0.01 & $\begin{array}{c}\downarrow 2(20 \%) \\
\uparrow 73\end{array}$ & $\begin{aligned} \downarrow & 1(5 \%) \\
& \uparrow 18\end{aligned}$ \\
\hline Chronic renal disease & 9 & 0.01 & 36 & 0.03 & 33 & 0.02 & 29 & 0.02 & 82 & 0.06 & $(811.1 \%)$ & $(202.7 \%)$ \\
\hline Haemophilia B & 1 & 0.00 & 1 & 0.00 & 1 & 0.00 & 1 & 0.00 & 2 & 0.00 & $\uparrow 1(100 \%)$ & $\uparrow 1(25 \%)$ \\
\hline Total & 129799 & 100.00 & 139634 & 100.00 & 136224 & 100.00 & 123580 & 100.00 & 136097 & 100.00 & $\begin{array}{l}\uparrow 6298 \\
(4.8 \%) \\
\end{array}$ & $\begin{array}{l}\uparrow 1575 \\
(1.2 \%) \\
\end{array}$ \\
\hline
\end{tabular}

Table 5: Average number of CDL conditions per patient, adjusted for age and gender

\begin{tabular}{|c|c|c|c|c|c|c|c|}
\hline Diagnosis & $\begin{array}{c}\text { Total } \\
\text { Number of } \\
\text { patients } \\
(2008-2012)\end{array}$ & Year & $\begin{array}{c}\text { Mean } \\
\text { (number of } \\
\text { CDL } \\
\text { conditions/pa } \\
\text { tients) }\end{array}$ & $\begin{array}{l}\text { Standard } \\
\text { Error }\end{array}$ & $\begin{array}{l}\text { Confidenc } \\
\text { e interval } \\
(95 \% \mathrm{Cl})\end{array}$ & $\begin{array}{c}\mathrm{p}- \\
\text { value }\end{array}$ & $\begin{array}{c}\text { Effect } \\
\text { size } \\
\text { (d-value) }\end{array}$ \\
\hline $\begin{array}{l}\text { All CDL } \\
\text { conditions }\end{array}$ & 1222444 & $\begin{array}{l}2008 \\
2009 \\
2010 \\
2011 \\
2012 \\
\end{array}$ & $\begin{array}{l}1.57 \\
1.68 \\
1.69 \\
1.72 \\
1.77 \\
\end{array}$ & $\begin{array}{l}0.00 \\
0.00 \\
0.00 \\
0.00 \\
0.00\end{array}$ & $\begin{array}{l}1.57-1.58 \\
1.67-1.68 \\
1.68-1.69 \\
1.71-1.72 \\
1.77-1.78 \\
\end{array}$ & 0.00 & 0.22 \\
\hline
\end{tabular}


beneficiaries as reported by the pharmaceutical benefit company used in this study. Similar increased burden of disease associated with chronic conditions was reported by a study conducted in twenty three different countries [8].

The current study reported higher prevalence of CDL conditions in female patients as compared to male patients since 2008 to 2012. Higher prevalence of non-communicable disease in females was also reported by other studies conducted in South Africa and Germany[9,10]. Findings from another study conducted in United States reported higher prevalence of co-morbid chronic conditions in females as compared to males [11].

The present study found an increase in CDL conditions proportionate to increase in age. This might be due to the fact that people live longer than before. According to a survey, one in twenty people were aged 65 years and older a century ago, however, the proportion was one in six in 2011 and it has been estimated that by 2050, the proportion will be one in four with chronic disease. This situation will lead to an increase risk of CDL conditions such as hypertension, hyperlipidaemia and diabetes associated with aging [8]. The results of conducted in the United States showed that prevalence of co-morbid chronic conditions increase with increase in age. Higher prevalence of non-communicable diseases in elderly was also reported by another study conducted in South Africa [12].

It has been estimated that deaths associated with all chronic diseases globally, may increase from $61 \%$ of the total disease burden in 2005 to $71 \%$ in 2030.On the other hand, deaths from cardiovascular disease and chronic respiratory disease may increase from 33 to $36 \%$ and 8 to $12 \%$ of the total disease burden by 2030 [8]. The findings of the current study revealed that chances of co-morbidities in individual patients with a CDL condition are high. Chronic renal disease was the only CDL condition with patients having an overall decrease in co-morbidity, although the average was still higher than the other conditions over the majority of the study period. This higher co-morbidity prevalence might be due to associated risk factors, such as hypertension, diabetes mellitus type 2 and obesity.

The high average number of co-morbid CDL conditions for coronary artery disease, may be attributed to diabetes mellitus type 2 and hyperlipidaemia, as being most commonly prevalent CDL condition [13]. The present study reported hypertension and hyperlipidaemia among the top three most prevalent chronic diseases. These results are in line with similar findings from another study conducted in South Africa [14]. Another study conducted in United States confirmed hypertension and hyperlipidaemia currently as the most prevalent chronic diseases [15].The increase in burden of chronic diseases will result in use of complex drug regimens which in turn will not only increase cost of therapy but will also promote issues related to unwanted side effects, drug-drug interactions, resistance and patient noncompliance.

\section{Limitations of the study}

The results of the study are based on the data from the medicine claims database of a nationally (South African) representative PBM company and might not be generalized to the entire country.

\section{CONCLUSION}

The prevalence of patients with CDL conditions along with risk of co-morbidity has been increasing with time in private healthcare sector of South Africa. Individual patients with any of the CDL conditions acquired on average at least one additional CDL co-morbidity (except for chronic renal disease), especially, when other CDL conditions were known as risk factors. Risk of increase co-morbidities with age and among different genders was prevalent. The current situation will easily overwhelm healthcare system as awareness regarding the magnitude and consequences of increased chronic disease burden as well as the capacity to respond appropriately with cost-effective measures is still low. Thus, this calls for more extensive research to recognise and accommodate the impact of the increased chronic disease burden to address this public health concern appropriately, especially in the private healthcare section of South Africa.

\section{ACKNOWLEDGEMENT}

We thank Dr. Suria Ellis from Statistical Consultation Services, North-West University, Potchefstroom Campus, for statistical support as well as Ms. Anne-Marie Bekker for administrative support regarding the database. The Pharmacy Benefit Management (PBM) company is thanked for providing the data used in this study. 


\section{DECLARATIONS}

\section{Conflict of Interest}

No conflict of interest associated with this work.

\section{Contribution of Authors}

The authors declare that this work was done by the authors named in this article and all liabilities pertaining to claims relating to the content of this article will be borne by them.

\section{REFERENCES}

1. Unwin N, Alberti K. Chronic non-communicable diseases. Ann Trop Med Parasitol, 2006; 100: 455-464.

2. Organization WH. Global health risks: mortality and burden of disease attributable to selected major risks. World Health Organization 2009.

3. Homer J, Hirsch G, Minniti M, Pierson M. Models for collaboration: How system dynamics helped a community organize cost-effective care for chronic illness. SYST DYNAM REV, 2004; 20: 199-222.

4. Mayosi BM, Flisher AJ, Lalloo UG, Sitas F, Tollman SM, Bradshaw $D$. The burden of non-communicable diseases in South Africa. The Lancet, 2009; 374: 934947.

5. Aikins Ad-G, Unwin N, Agyemang C, Allotey P, Campbell C, Arhinful D. Tackling Africa's chronic disease burden: from the local to the global. Globalization and Health, 2010; 6: 5

6. Doherty J, McLeod H. Medical schemes: framework for transformation. SAHR, 2002: p. 41-66.

7. Schemes. CfM. Annual report for 2010/2011. In: ed.^eds. Council for Medical Schemes. Pretoria, 2011.
8. Abegunde DO, Mathers $C D$, Adam T, Ortegon M, Strong $K$. The burden and costs of chronic diseases in lowincome and middle-income countries. The Lancet, 2007; 370: 1929-1938.

9. Phaswana-Mafuya N, Peltzer K, Chirinda W, Musekiwa A, Kose Z, Hoosain E, Davids A, Ramlagan S. Selfreported prevalence of chronic non-communicable diseases and associated factors among older adults in South Africa. Glob Health Action, 2013; 6.

10. van den Bussche $H$, Koller $D$, Kolonko $T$, Hansen $H$, Wegscheider K, Glaeske G, von Leitner E-C, Schäfer I, Schön G. Which chronic diseases and disease combinations are specific to multimorbidity in the elderly? Results of a claims data based cross-sectional study in Germany. BMC public health, 2011; 11: 101.

11. Paez KA, Zhao L, Hwang W. Rising out-of-pocket spending for chronic conditions: a ten-year trend. HEALTH AFFAIR, 2009; 28: 15-25.

12. Phaswana-Mafuya N, Peltzer K, Chirinda W, Musekiwa A, Kose Z, Hoosain E, Davids A, Ramlagan S. Selfreported prevalence of chronic non-communicable diseases and associated factors among older adults in South Africa. Global health action, 2013; 6.

13. Nobili A, Garattini S, Mannucci PM. Multiple diseases and polypharmacy in the elderly: challenges for the internist of the third millennium. JOC, 2011; 1: 28-44.

14. McLeod H, Rothberg A, Pels L, Eekhout S, Mubangizi $D B$, Fish T. The Costing of the Proposed Chronic Disease List Benefits in South African Medical Schemes in 2001. Centre for Actuarial Research, University of Cape Town, 2002.

15. Ornstein SM, Nietert PJ, Jenkins RG, Litvin $C B$. The prevalence of chronic diseases and multimorbidity in primary care practice: a PPRNet report. JABFM, 2013; 26: $518-524$ 